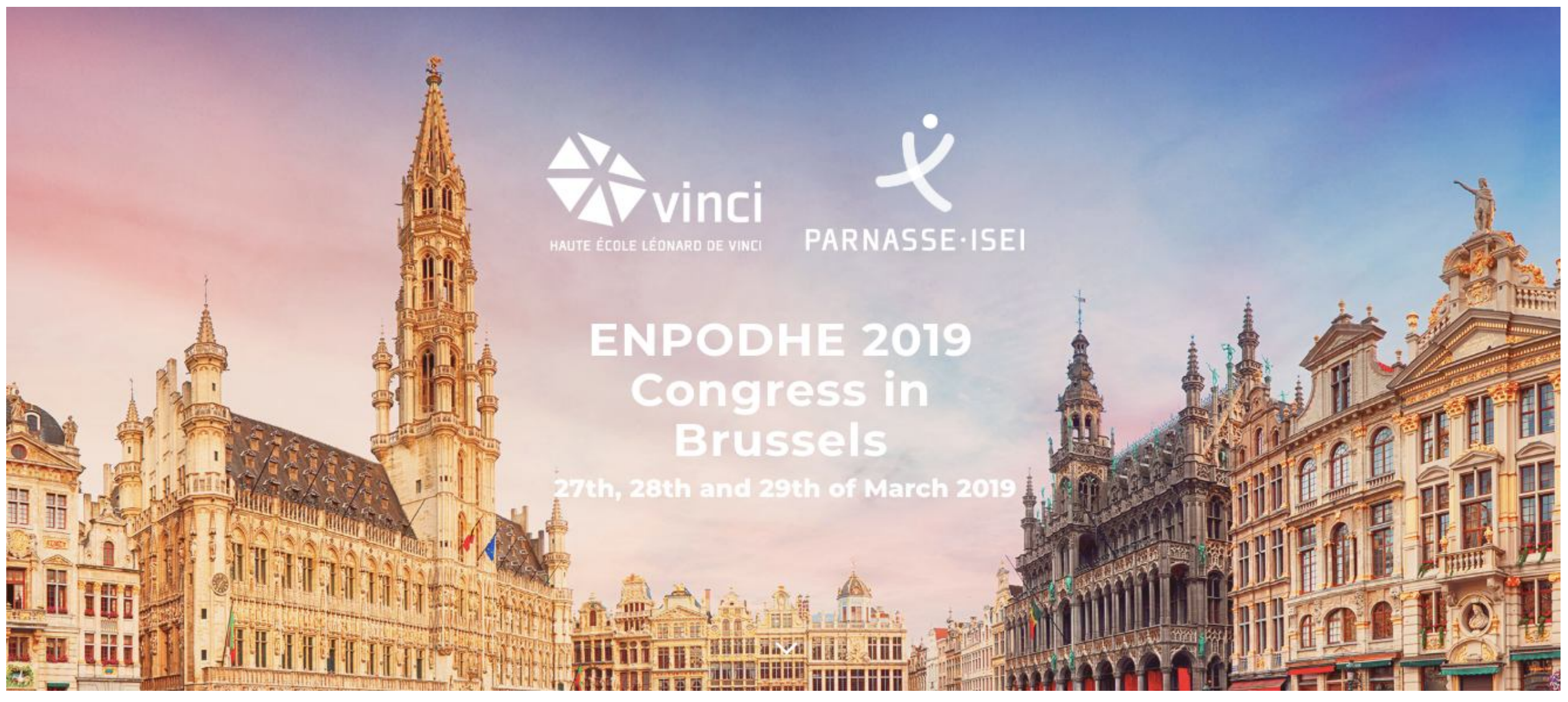

\title{
The influence of childhood obesity on spatio-temporal gait parameters
}

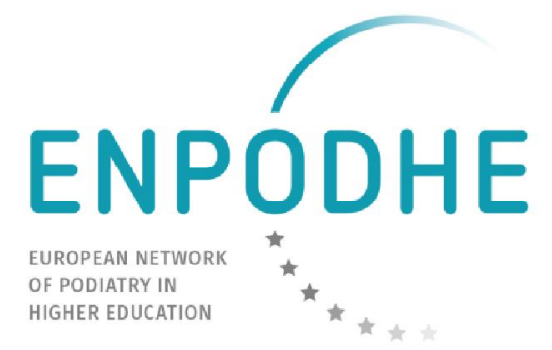

Author: Montes Alguacil, Jesús 


\section{INTRO}

Overweight and obesity in 6-12 year old children in Switzerland

Micbael B. Zimmermann , Carolyn Gübeli', Clandia Pïntener , Luciano Molinart

a Laboratory for Human Nutrition, Institute for Food Science and Nutrition,

Swiss Federal Institute of Technology, Zurich, Switzerland

"Institute for Pharmaceutical Science, Swiss Federal Institute of Technology, Zurich, Switzerland

Department of Growth and Development, University Children's Hospital, Zurich, Switzerland
Global prevalence and trends of overweight and obesity among preschool children ․ㅏㅇ

Mercedes de Onis Monika Blössner, Elaine Borghi

The American Journal of Clinical Nutrition, Volume 92, Issue 5, 1 November 2010, Pages 1257-1264, https://doi.org/10.3945/ajcn.2010.29786 


\section{INTRO}

Changes of intersegment angular motion of the body during gait. Strutzenberger G. 2011; Shultz SP. 2014; Mahaffey R. 2016

Pogression of angular deformities in varus/valgus of the knee. Mc Millan AG. 2010

An increased risk of osteoarthritis in adulthood. Strutzenberger G. 2011; Shultz SP. 2014-Clin.

A less walking stability in obese children than those with normal weight. Yan S. 2013

Obese children need to produce more energy in the joints of lower limbs. Shultz SP. 2014

Other... 


\section{INTRO METHODS RESULTS DISGUSSION GONGLUSION}
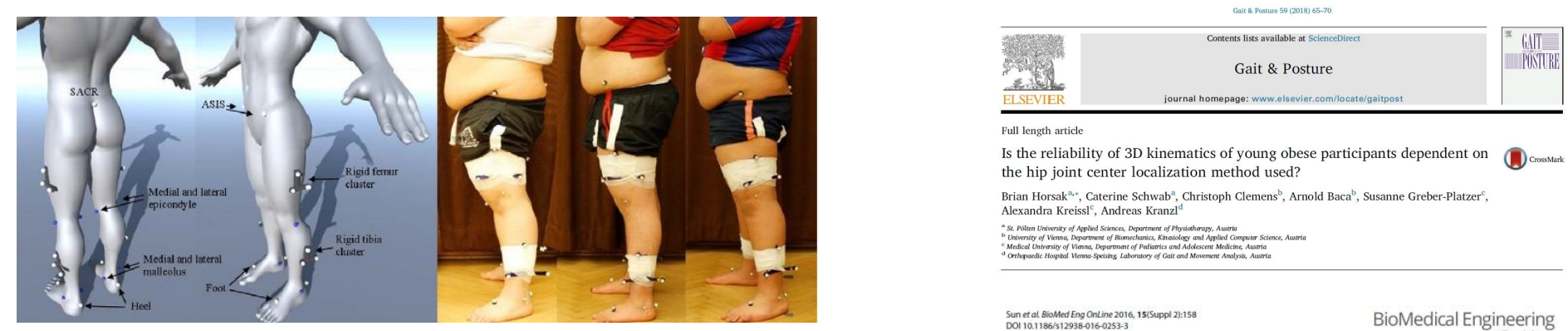
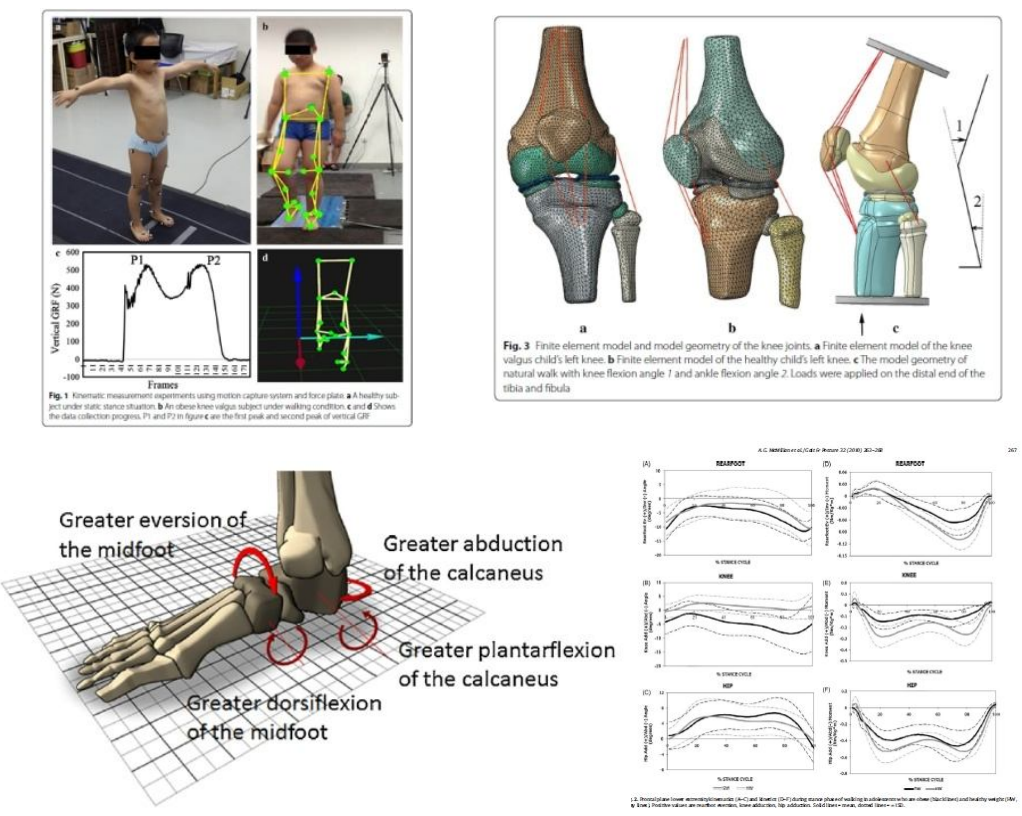
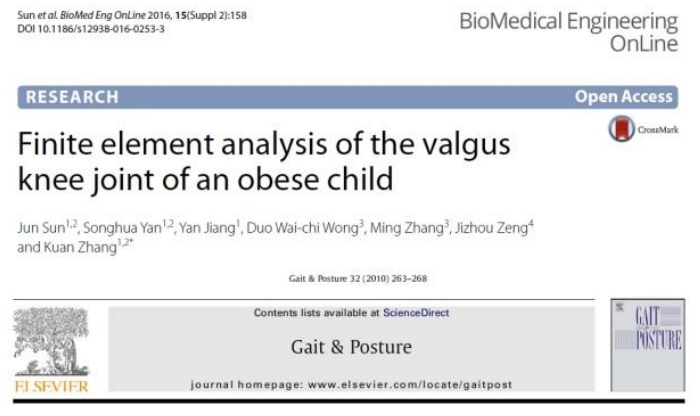

Sagittal and frontal plane joint mechanics throughout the stance phase of walking
in adolescents who are obese

A.G. McMillan a., A.M.E. Pulvera, D.N. Collier b. D.S.B. Williams

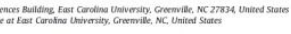

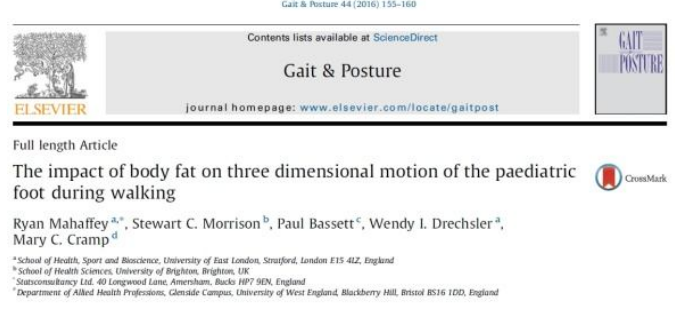




\section{Childhood Obesity and Gait Parameters}

\section{INTRO}

Optogait Photoelectric System
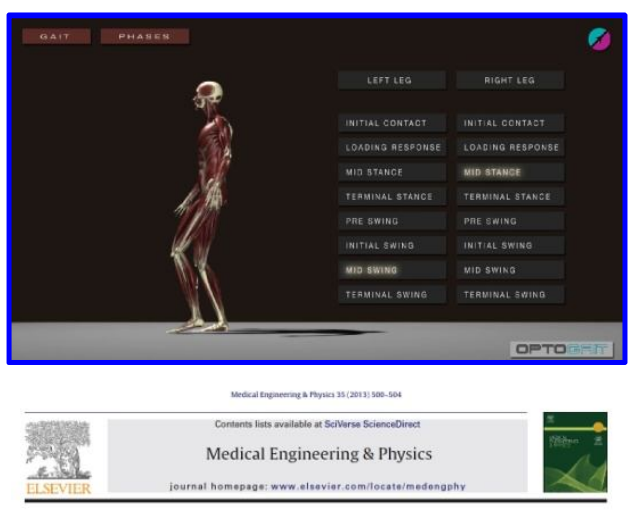

Validity of the Optogatit photoelectric systen for the asessnent of

spatiotemporal gait parameters
Karin Lienhard, David Schneider, Nicola A Maffuluetrit

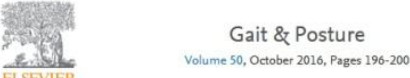

$\frac{\pi}{1.5 \times 12}$

Reliability of the OptoGait portable

photoelectric cell system for the quantification

of spatial-temporal parameters of gait in

young adults
CIRCUMSTANCES OF THE STUDY

\section{CHILDREN}

WALKING

OVERGROUND
AT SELF-

SELECTED

VELOCITY 


\section{METHODS}

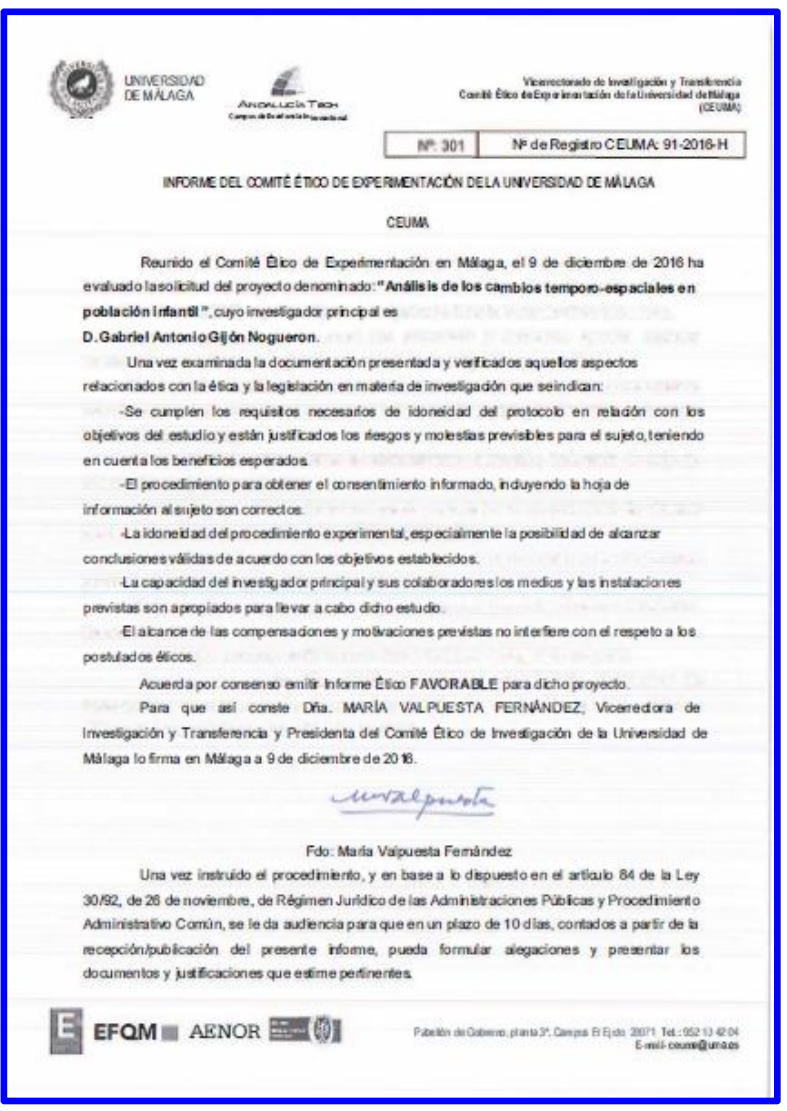

\section{Ethical Issues:}

Parents provided signed consents.

Ethics Committee of the University of Malaga. CEUMA 91/2016-H)

Declaration of Helsinky. 
METHODS

\section{Participants:}

$\mathrm{N}=238$ participants, 7 to 11 years old

Inclusion/Exclusion criteria

Primary schools. 


\section{Childhood Obesity and Gait Parameters}

\section{METHODS}

\section{Data Collection:}

Anthropometrics variables.

Body Mass Index. Classification. Sobradillo 2004

\begin{tabular}{|c|c|c|c|c|c|}
\hline Age $(y)$ & $n(\%)$ & Underweight ( $\mathrm{n} \%$ ) & Normalweight ( $/ \%$ ) & Overweight $(\mathrm{n} / \%)$ & Obese $(\mathrm{n} / \%)$ \\
\hline $\begin{array}{l}\mathbf{3} \\
\end{array}$ & $35 / 1470$ & m & 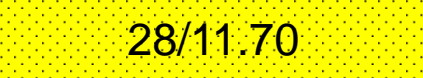 & 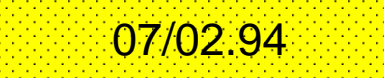 & 'ڤ \\
\hline 8 & $45 / 1890$ & $1 / 0,42$ & $25 / 1050$ & $06 / 0252$ & $13 / 0546$ \\
\hline 9 & $44 / 1848$ & 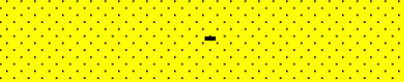 & $22 / 0924$ & 110462 & $11 / 0462$ \\
\hline 10 & $57 / 2394$ & $2 / 084$ & $32 / 1344$ & $09 / 0378$ & $14 / 0588$ \\
\hline 11 & $57 / 23.94$ & 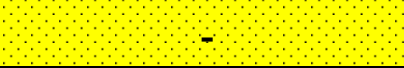 & $29 / 1218$ & $12 / 0504$ & $16 / 0672$ \\
\hline
\end{tabular}


METHODS

\section{Data Collection:}

Spatio-temporal variables were collected by Optgait system

\section{Stance phase Swing phase Single support Double support}

Step length Step time Load response phase Pre-swing phase

Contact phase Foot flat phase Propulsive phase Gait cycle Stride length Speed Acceleration Cadence Total distance. 
METHODS

\section{Statistical Analysis:}

Exploratory analysis by Kolmogorov-Smirnov

Bivariante analysis by Student`s Test

Multivariante by ANOVA

$\underline{\text { In addition... }}$

Levene Test

Browne-Forsythe Test (Robustness)

Bonferroni Test (Post-hoc) 


\section{Childhood Obesity and Gait Parameters}

\section{INTRO METHODS RESULTS DISGUSSION GONGLUSION}

Anthropometric characteristics of the sample by gender

\begin{tabular}{|c|c|c|c|c|c|c|c|c|}
\hline & & \multirow[b]{2}{*}{$\mathrm{Mean}$} & \multicolumn{2}{|c|}{$95 \%$ Confidence lnterval } & \multirow[b]{2}{*}{$\mathrm{SD}$} & \multirow[b]{2}{*}{$\mathrm{Mr}$} & \multirow[b]{2}{*}{$\mathrm{Ma}$} & \multirow[b]{2}{*}{$\mathrm{ser}$} \\
\hline & & & ower & Upper & & & & \\
\hline \multirow[t]{2}{*}{$A g e$ years) } & Female & 929 & 903 & 9,55 & 140 & 7 & 1 & 01310 \\
\hline & Male & 919 & 894 & 943 & 138 & 7 & 11 & 0,1240 \\
\hline \multirow[t]{2}{*}{$\mathrm{Height}(\mathrm{m})$} & Female & 13701 & 13454 & 13948 & 01332 & 110 & 1,69 & 0,0124 \\
\hline & $\mathrm{Male}$ & 3539 & 13341 & 13736 & 0,1111 & 109 & 159 & 00099 \\
\hline \multirow[t]{2}{*}{ Weight $(\mathrm{kg})$} & Female & 39,30 & 3692 & 41,67 & 12,78 & 19 & 82 & 197 \\
\hline & $\mathrm{Male}$ & 38,55 & 36,30 & 40,81 & 1268 & 19 & 86 & 139 \\
\hline Body Mass & Female & 20,41 & 19,69 & 2113 & 3,89 & 1475 & 3255 & 03646 \\
\hline Index $\left(\mathrm{kg} / \mathrm{m}^{2}\right)$ & Male & 20,54 & 19,77 & 21,31 & 4,33 & 13,7 & 36,18 & 0,3895 \\
\hline
\end{tabular}




\section{RESULTS}

No statistical differences related to spatial parameters.

Temporal parameters with $\mathrm{p}<0.05$ in obese and overweight children.

\section{Stance phase Swing phase Single support Double support}

Step length Step time Load response phase Pre-swing phase

Contact phase Foot flat phase Propulsive phase Gait cycle Stride length Speed Acceleration Cadence Total distance. 


\section{Childhood Obesity and Gait Parameters}

\section{RESULTS}

No statistical differences related to spatial parameters.

Temporal parameters with $\mathrm{p}<0.05$ in obese and overweight children.

\section{Stance phase}

\section{Load response phase Pre-swing phase}


INTRO METHODS RESULTS DISGUSSION GONGLUSION

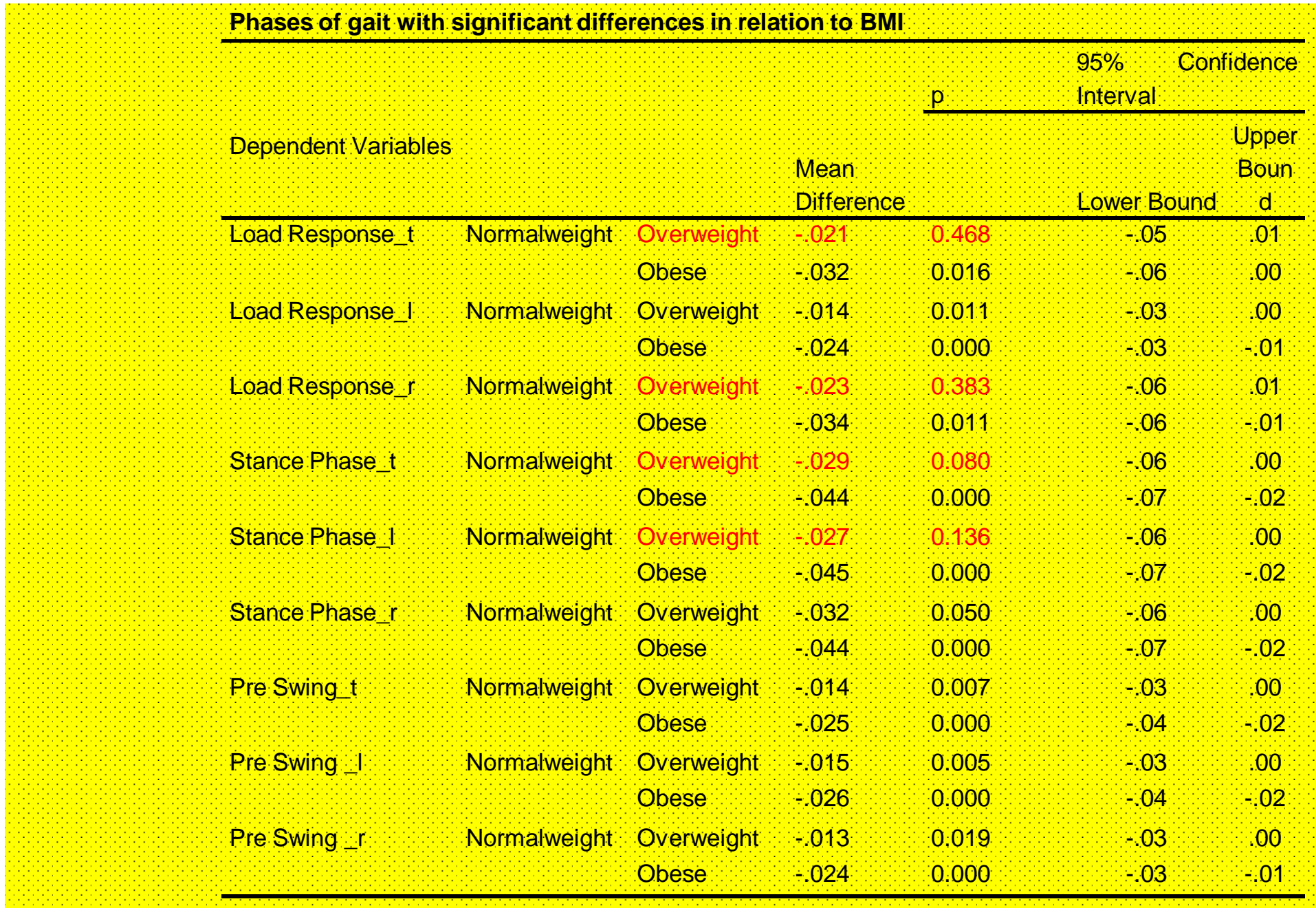




\section{DISCUSSION}

\section{PREVIOUS STUDIES. SIGNIFICANT RESULTS:}

3-D Analysis and Force platforms:

Stance phase, Step width, Pre-swing phase

Mahaffey R. 2016, Mc Millan AG. 2010, Cimolin V. 2015, Huang L. 2013, Hung Y-C. 2013

Photoelectric systems:

Stance phase, Pre-swing phase

Beulertz J. 2016, Galli M. 2015 
INTRO METHODS RESULTS DISCUSSION

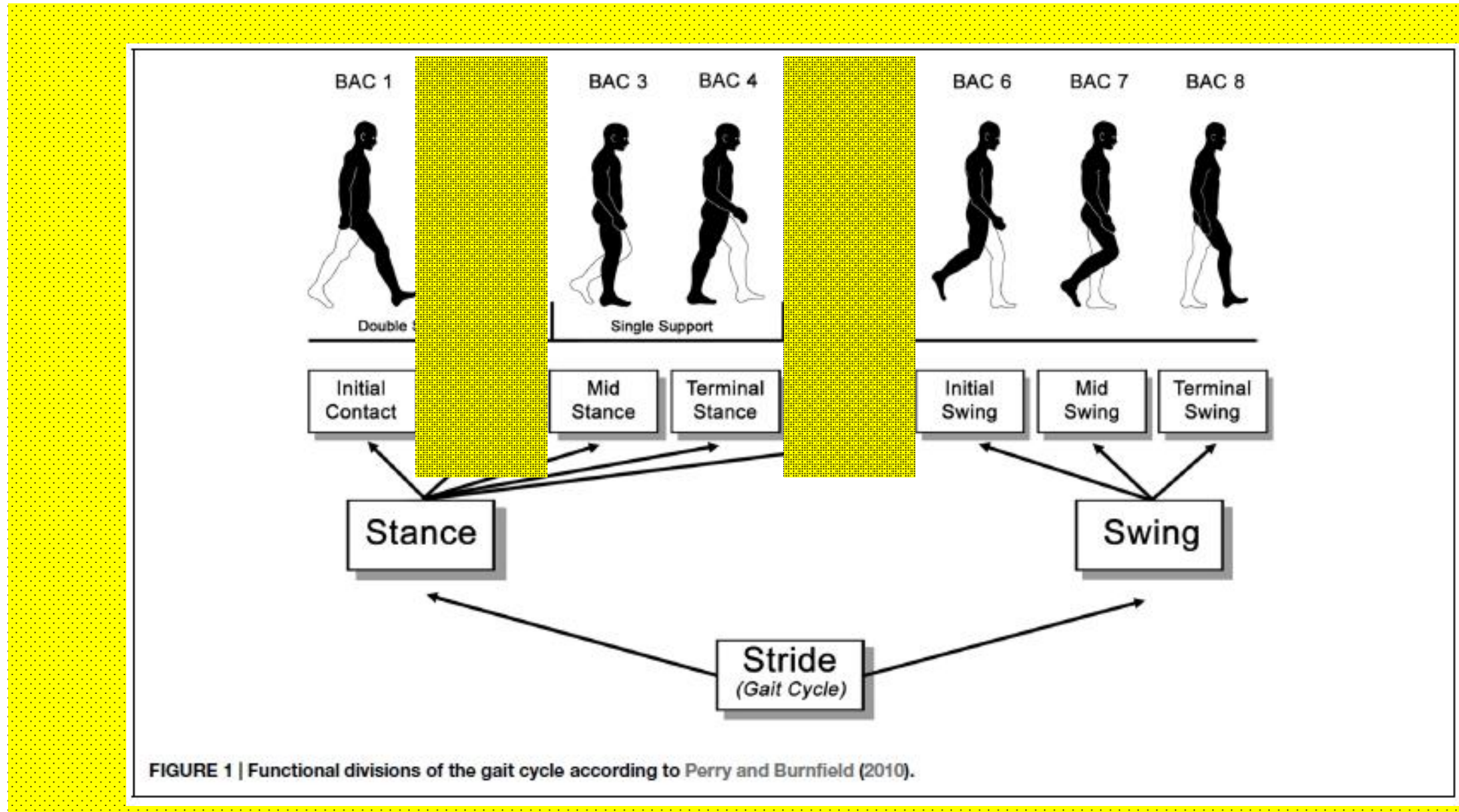


Childhood Obesity and Gait Parameters

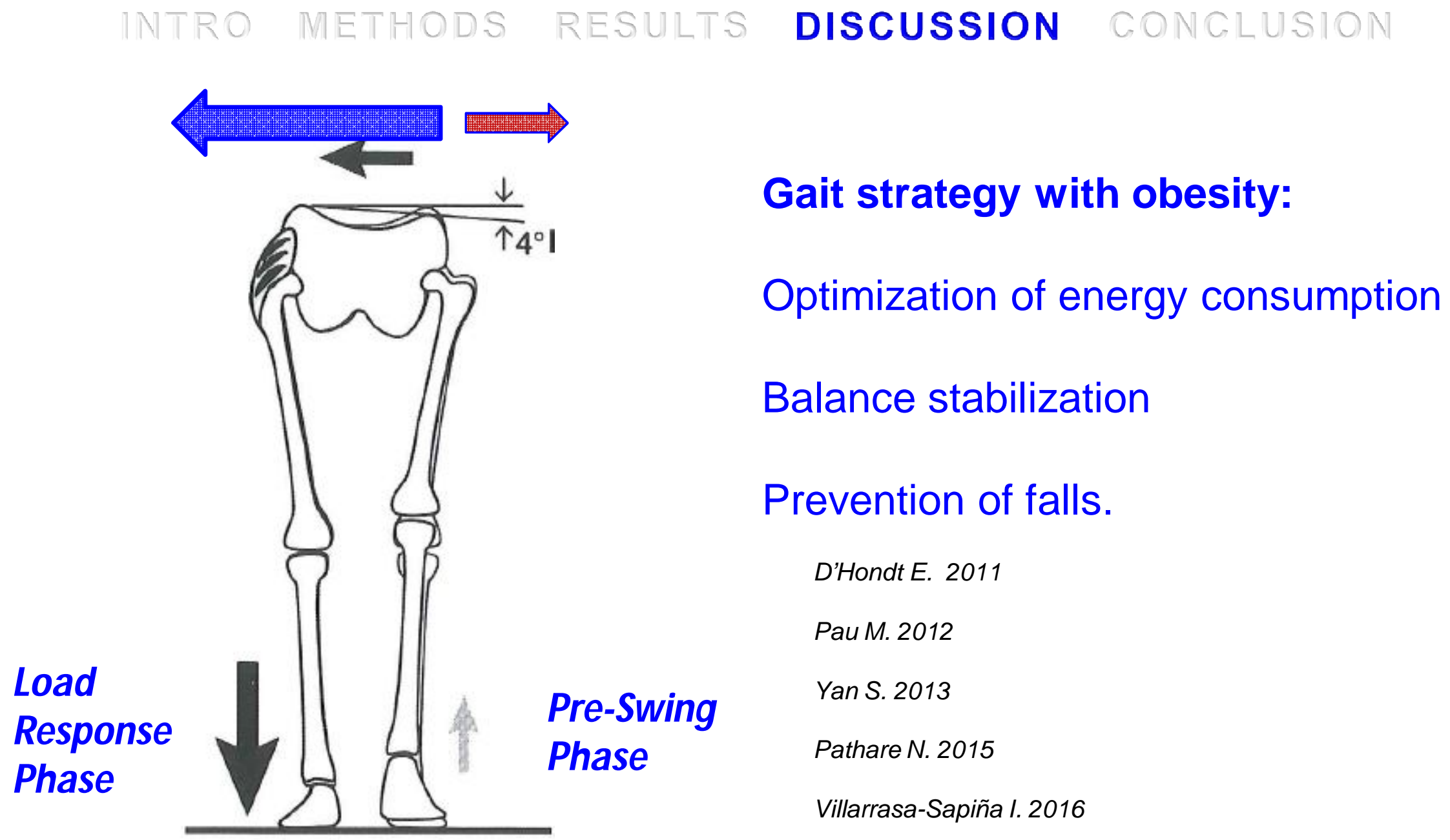


Obesity influences Spatio-temporal Gait Parameters of Children

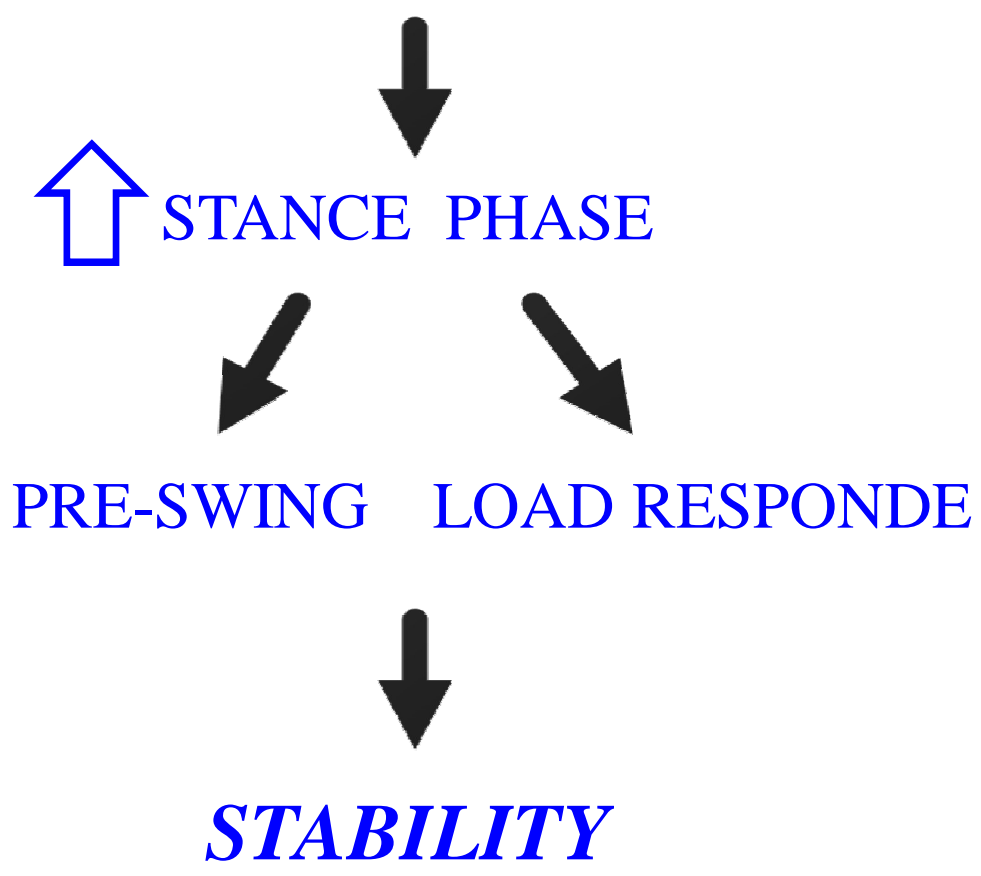


Thank you! 
